\title{
Approach to perinatal mental health and child abuse prevention in Japanese prefectural health centers
}

\author{
Kafumi Sugishita ${ }^{1,2^{*}}$, Kayoko Kurihara ${ }^{1}$, Shiho Murayama ${ }^{1}$, Kiyoko Kamibeppu $^{1}$ \\ ${ }^{1}$ Department of Family Nursing, Graduate School of Medicine, The University of Tokyo, Tokyo, Japan; \\ *Corresponding Author: kafumis@med.nagoya-cu.ac.jp \\ ${ }^{2}$ Reproductive Health Nursing/Midwifery, Graduate School of Nursing, Nagoya City University, Nagoya, Japan
}

Received 10 February 2013; revised 20 March 2013; accepted 5 April 2013

Copyright (C) 2013 Kafumi Sugishita et al. This is an open access article distributed under the Creative Commons Attribution License, which permits unrestricted use, distribution, and reproduction in any medium, provided the original work is properly cited.

\section{ABSTRACT}

The aim of this study was to conduct a nationwide survey in Japan of prefectural health centers, which were responsible for providing guidance to municipalities. The survey was performed in order to clarify the following issues: 1) the current level of support provided by prefectural centers for pre- and post-natal mental health; 2) the structures in place for providing consultation services for an "unwanted pregnancy" and the support available for high-risk cases; and 3) the advice available on postpartum maternal psychological screening, and interpretation of results of such screening. Questionnaires were sent by post to $\mathbf{3 9 4}$ prefectural health centers, of which 277 (70.3\%) responded. A total of $32 \%$ of prefectural health centers confirmed that they had offered support to high-risk cases during pregnancy, and $72 \%$ had offered support post-partum. Regarding offering support to high-risk mothers, those prefectural health centers that did provide consultation services (n = 59) reported providing introductions and information about available facilities $(P<0.001)$ and conducting case conferences $(P<0.002)$. This was significantly different than prefectural health centers that did not provide consultation services $(n=198)$. At the prefectural health centers that "follow up on" the results of the mental health screening, psychiatry consultations were reported twice as often as the prefectural health centers that did "not follow up on" the results of mental health screening. These findings indicate that childcare support systems for postpartum mental health and the prevention of child abuse were established. However, the lack of prenatal health and support systems for the prenatal pe- riod remains an issue.

Keywords: Child Abuse Prevention; Perinatal Mental Health; Prefectural Health Centers; Municipal Health Centers

\section{INTRODUCTION}

The number of cases of child abuse handled by child consultation centers in Japan was 44,210 in 2009, and has been increasing each year. In addition, cases of abuse resulting in death are recorded at a rate of one per week. Relevant organizations must therefore respond promptly to cases of child abuse. Sukoyaka Oyako 21 is a national Japanese plan to improve the health of mothers and children from 2001 to 2014. The plan consists of four main goals, the two we will consider are: 1 ) to reduce the occurrence rate of postpartum depression; and 2) to reduce the number of fatal cases of child abuse. An intermediate evaluation of the plan was conducted by the Ministry of Health, Labour and Welfare [1]; however, the incidence of postpartum depression could not easily be assessed due to participant and regional differences. The effects of a manual on postpartum mental health and child-rearing recently produced by the Japanese Ministry Scientific Research Group (2004-2006) are unclear. Mortality due to child abuse in Japan remains unchanged.

Another review of the plan presented in June 2007 indicated that in fatal cases of child abuse: 1) $40 \%$ of cases involve children less than 1 year old; 2) unwanted pregnancy is a risk factor; 3) maternal emotional conflict during pregnancy is a risk factor; and 4) $70 \%$ of cases are from relatively poor families [2].

In the 1990s, the use of home visits during pregnancy was studied in several western countries [3,4]. Home visits were conducted with several strategies geared towards unwanted pregnancies, adolescent pregnancies and 
pregnancies in low-income groups: factors that were found to contribute to child abuse. These home visits were primarily geared towards groups prone to child abuse, i.e., women with unwanted pregnancies, pregnant adolescents and low-income families [5-7]. On the other hand, several studies have been made on the association between unwanted pregnancies and perinatal depression [8-10]. In Japan, a home visiting program, Boshi-AiikuKai, has been sponsored by the Mother-Child Nursing Association since the 1930s. More recently, a universal (population) home visiting service project, KonnichiwaAkachan-Gigyo, was founded in 2007 for the purpose of preventing infant abuse. Participants are automatically enrolled in the service when they report their pregnancy to the local government office or when they register their child's birth.

In 2008, a community health and medical institution cooperation system was created by the Ministry of Health, Labour and Welfare. In this system, local community health institutions cooperated with hospitals in order to support pregnant women who are prone to child abuse. As part of this system, when either a medical institution or a municipal health center performs a health check-up during pregnancy, information regarding high-risk pregnant women is exchanged between the institutions. A particular emphasis is focused on abuse prevention by early identification of high-risk families during pregnancy.

Until recently in Japan, prefectural health centers played a front-line role in child abuse prevention. However, enforcement of the Community Health Law has shifted a part of those responsibilities concerning the prevention of child abuse to municipalities, or wards. Each of the 47 prefectures in Japan contains between 23 and 50 municipalities. They are now responsible for consultation and management of difficult cases (high-risk mothers) as well as universal (population-based) heath concerns. Based on their past experiences, prefectural health centers must provide educational support for municipalities. Such support for municipal system development includes planning, research, surveys and training programs for mother-child-health leaders [11].

Previous research into the prevention of child abuse in prefectural health centers revealed that infant consultation services, utilization of parenting groups, and collaboration with municipal health centers are effective first steps for the prevention of child abuse [12]. A study that focused on the role of prefectural health centers and community health nurses who support premature infants identified the benefits of collaboration between organizations [13]. Another study reported that collaboration between prefectural health centers and medical institutions was important for the development of child abuse prevention programs [14]. Such collaboration has contrib- uted to the development and distribution of an abuse factor check-list [15]. Prefectural health centers have also attempted to systemize community health activities and have created and evaluated an infant abuse riskassessment index [16].

Previous studies have revealed that child abuse prevention is very important for the community. However, an effective approach to the prevention of child abuse that incorporates perinatal mental health, unwanted pregnancies, and postpartum depression has not been developed. The aims of the present study were: 1) to clarify the present situation regarding support in Japanese prefectural health centers for improving perinatal mental health in order to prevent child abuse; 2) to explore the association between the degree of consultation during unwanted pregnancies and support for high-risk mothers; and 3) to clarify the present situation regarding education of perinatal mental health screening and understanding of the results of such screening in prefectural health centers.

\section{METHODS}

\subsection{Subjects}

An inventory survey was conducted by mail with 394 prefectural health centers in Japan from November 2007 to February 2008.

\subsection{Questionnaire}

The questionnaire was prepared with reference to previous studies in the Journal of Health and Welfare Statistics of Japan [17-19], Maternal and Child Health Statistics of Japan [20,21], and guidelines prepared by the Ministry of Health, Labour and Welfare regarding how to intervene in child abuse and neglect [22]. The delivery address for all of the questionnaires was unified to "the person responsible for support for maternal and child health activities in the municipality”. Also advice was obtained from staff members of the Ministry of Health, Labour and Welfare. Subjects who returned answered questionnaires were considered to have consented to participate. Institutional privacy was maintained.

\subsection{Data Analysis}

Descriptive analyses were conducted in order to clarify the present situation regarding support in Japanese prefectural health centers for improving perinatal mental health in order to prevent child abuse. To explore the association between the degree of consultation during unwanted pregnancies and support for high-risk mothers, the Fisher's exact test was used to compare outcomes between groups with and without consultation services. To clarify the association between prefectural health centers' understanding of mental health screening results 
and postpartum support with municipalities, the Fisher's exact test was used to compare outcomes between understanding and not understanding groups. SPSS $16.0 \mathrm{~J}$ for Windows was used to analyze the description statistics. The level of statistical significance was set to $\mathrm{P}<0.05$.

\section{RESULTS}

\subsection{Demographics}

Responses were received from 277 prefectural centers, for a collection rate of $70.3 \%$. Among the respondents, 267 (96.4\%) were public health nurses (front-line staff). The population of the catchment area in $35.7 \%$ of the health centers was less than 100,000 people, and $26.4 \%$ had population between 100,000 and 200,000. Among all works of the prefectural health centers which participated, the mean ratio of the maternal and child health business was $18.8 \% \pm 16.8 \%$.

\subsection{Support during the Prenatal Period}

Regarding referrals for difficult prenatal cases, 83\% of the prefectural health centers reported that there is a system for consultation from the municipalities. The two kinds of municipal prenatal support and consultation provided by prefectural health centers were direct support and indirect support. Thirty-two percent of prefectural health centers reported having supported difficult prenatal cases in 2006.

Methods of direct support for consultation of difficult cases from the municipalities included home visits in $68 \%$ and both interview consultation and telephone consultation in $53 \%$ of prefectural health centers. Methods of indirect support for consultation of cases from the municipalities included home visits by municipal public health nurses in $78 \%$ of public health centers. The number of cases that were supported through the postpartum period in each prefectural center ranged from 0 to 18 cases in 2006.

Prefectural health center respondents reported that home visits by public health nurses of the municipality were most desirable for various types of pregnant women. On the other hand, prefectural health centers responded that home visits by prefectural health center nurses were most suitable for pregnant women with mental disorders, pregnant women with a history of abusing a child, and pregnant women with a history of having been abused (Figure 1).

\subsection{Consultation Services for Women with Unwanted Pregnancies}

Regarding consultation for women with unwanted pregnancies, $22.9 \%$ of prefectural health centers responded that they provided consultation services. A total of $64 \%$ of prefectural health centers responded that while women's health support services are valuable, they had no plans to enact such services in the near future. Regarding support for high-risk mothers, prefectural health centers that did provide consultation services $(n=59)$

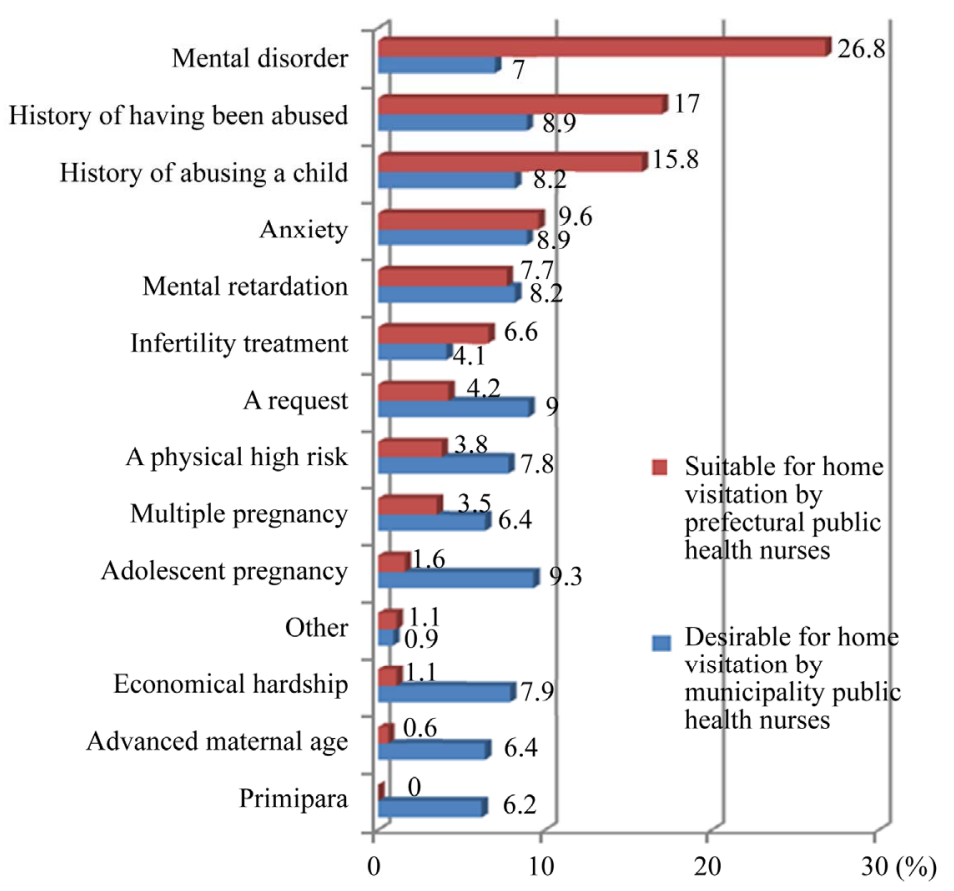

Figure 1. "Pregnant women for whom a visit from a prefectural public health nurse is suitable" and "Pregnant women for whom a visit from a municipal public health nurse is desirable”. 
reported providing introductions to associated facilities $(\mathrm{P}<0.001)$, conducting case conferences $(\mathrm{P}<0.002)$, and conducting network meetings $(\mathrm{P}<0.003)$; all significant compared to prefectural health centers that did not provide consultation services $(n=198)$. Concerning indirect support, prefectural health centers that provided consultation services conducted more kinds of support compared to prefectural health centers that did not provide consultation services (Table 1). This service had nothing to do with support for difficult postpartum cases. However, prefectural health centers that did provide consultation services offered child-rearing self-help groups such as Mother-Child Groups (MCG) more often than prefectural health centers that did not provide consultation services.

\subsection{Postpartum Support}

Regarding referrals for difficult postpartum cases, $95.5 \%$ of the prefectural health centers responded that there is a system for consultation from the municipalities, and $71.6 \%$ of prefectural health centers reported having supported difficult postpartum cases in 2006.

Concerning methods for direct support of postpartum consultation cases from the municipalities, $78.9 \%$ of prefectural health centers responded that they provided home visits, 56.8\% provided telephone consultation, $55.6 \%$ provided interview consultation, and $52.8 \%$ provided introduction to associated facilities.

Concerning methods for indirect support of postpartum consultation cases from the municipalities, $81.5 \%$ of prefectural health centers responded that they provided home visits by municipal public health nurses, 54\% conducted network meetings or explain about the network meeting, and $49.7 \%$ introduced municipal public health nurses to associated facilities. Prefectural health centers reported that home visits by the municipal public health nurses were more desirable subjects for various reasons. Prefectural health centers reported that home visits by prefectural public health nurses were more suitable for postpartum women with mental disorders, low-birthweight infants, postpartum women with a history of abuse, and women with postpartum depression (Figure 2).

\subsection{Postpartum Mental Health Screening}

Regarding whether prefectural health centers encouraged the use of a designated method for screening postpartum mental health, $40.8 \%$ responded that they had no special guidance, $29.2 \%$ used the 3 -set questionnaire

Table 1. Difference in support provided by prefectural health centers for women with unwanted pregnancies depending on the provision of prenatal and postpartum consultation services.

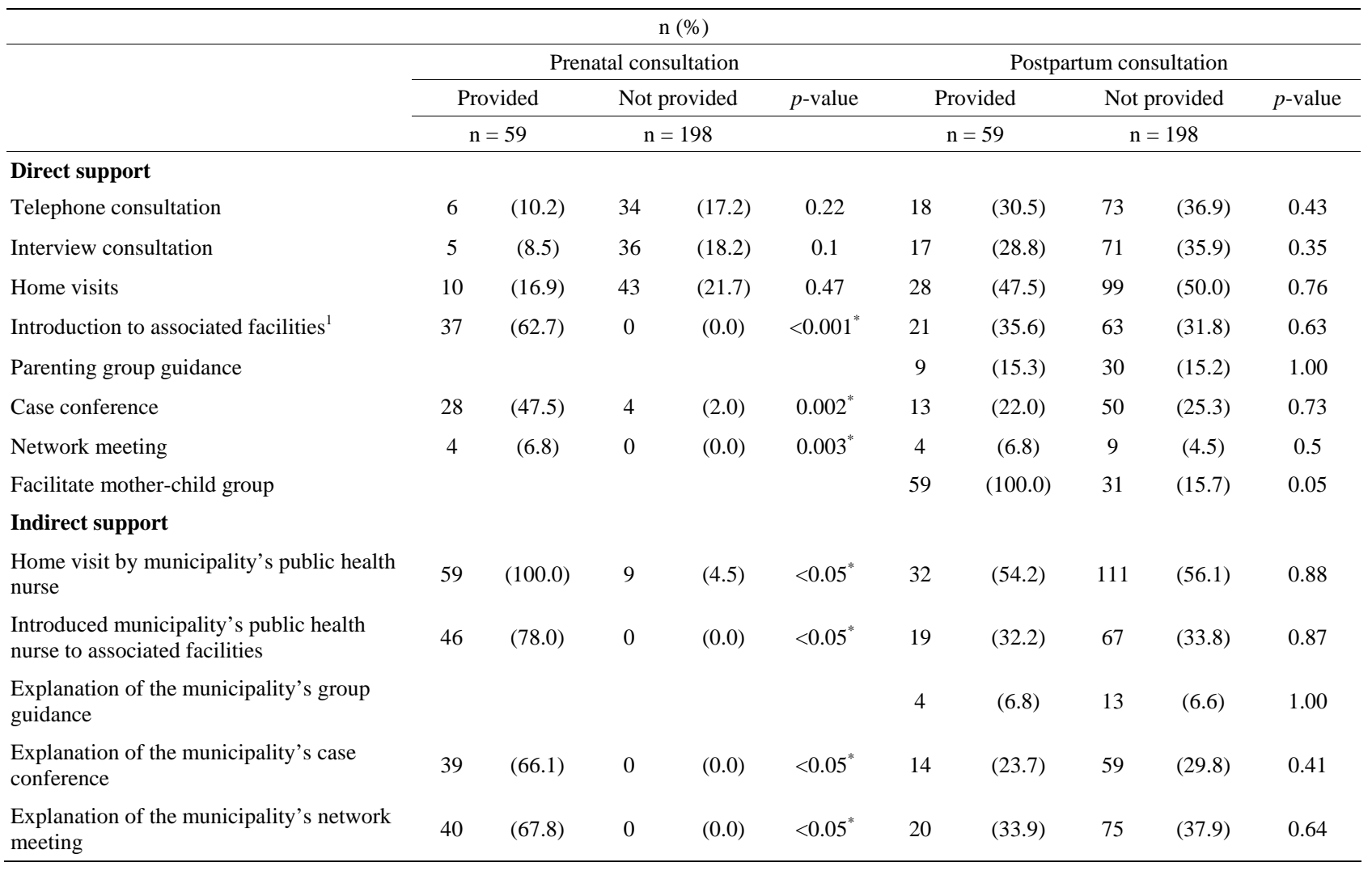

${ }^{1}$ Child abuse consultation center, hospital, school, etc; Fisher's exact test ${ }^{*} \mathrm{P}<0.05$. 


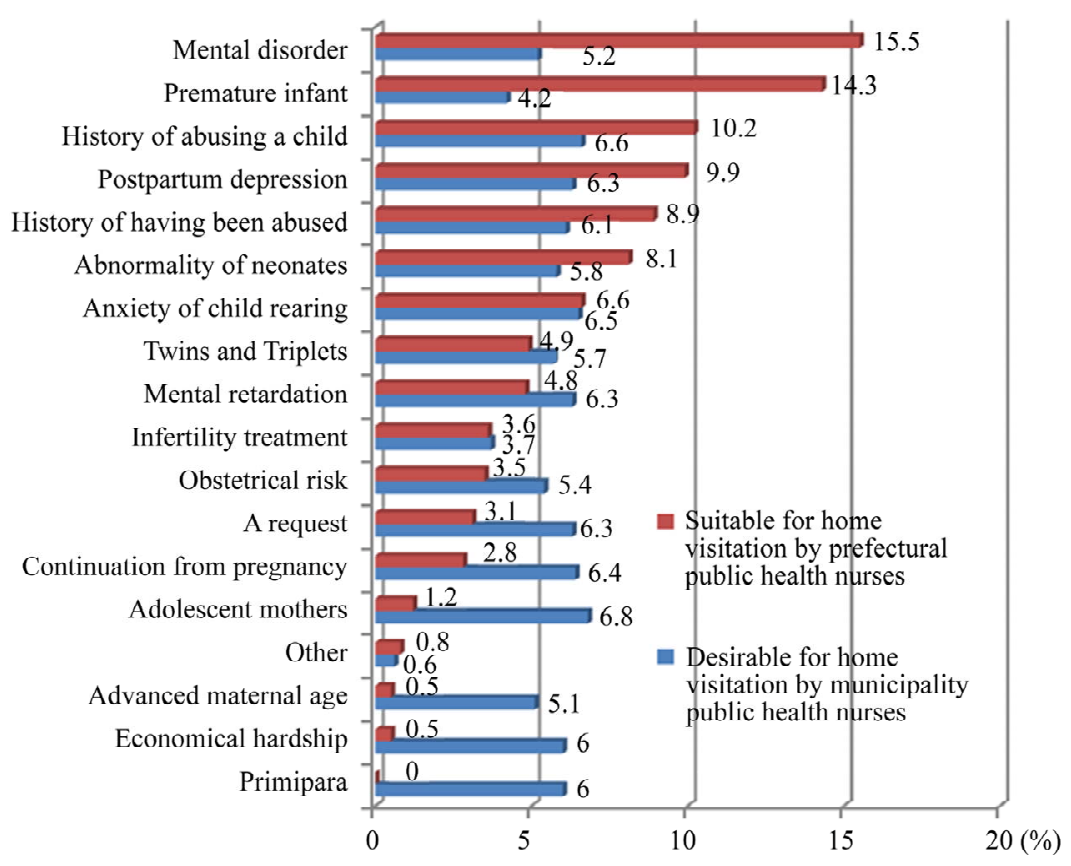

Figure 2. "Postpartum women for whom a visit from a prefectural public health nurse is suitable" and "Postpartum women for whom a visit from a municipal public health nurse is desirable".

(Abuse risk assessment [23], Edinburgh Postpartum Depression Scale [24] and Mother-to-Infant Bonding Scale [25] and 19.8\% used the Edinburgh Postpartum Depression Scale). The proportion of prefectural health centers that reported understanding the results of mental health screening of postpartum mothers was $50.5 \%$. At the prefectural health centers that understood the results of the mental health screening, psychiatry consultations were reported twice as often as the centers that did not understand the results of mental health screening (Table 2).

Concerning methods of direct support for high risk mothers, compared to prefectural health centers that did not understand mental health screening results $(n=127)$, significantly more prefectural health centers that understood mental health screening results $(n=136)$ provided parenting group guidance $(\mathrm{P}<0.001)$ and case conferences $(P<0.031)$ (Table 3). Concerning indirect support, prefectural health centers that understood mental health screening results provided more types of support compared to prefectural health centers that did not understand mental health screening results (Table 3).

\section{DISCUSSION}

\subsection{Prenatal Support}

Among Japanese prefectural health centers, $80 \%$ or more of cases were referrals corresponding to consultation from municipalities, but they actually supported only $30 \%$ of cases. Consultation cases from municipalities were pregnancies at high risk for child abuse.
Prefectural health centers utilize pregnant women information sheets and early child-rearing flow charts [23], as it is necessary to systematically gather information, request support and report cases between prefectural health centers and municipalities. While municipalities and prefectural health centers commonly recognize this need, it is thought that more concrete and mutual support is possible.

Direct and indirect support during the prenatal period most commonly took the form of home visits, which were conducted to support the daily life-based needs of pregnant women.

In western countries, several studies have been made on the efficacy of home visits during the prenatal period. In particular, home visits as a tool for the prevention of child abuse for high-risk mothers (such as a low-income or single mothers) has been reported [24,25]. In Japan, home visits are made for various kinds of difficult pregnancies. Most support for which prefectural health centers wanted municipalities to be careful included psychological support and information. Ideally, this support should continue until the postpartum period.

Prefectural health centers reported considering home visits from the municipalities to be most desirable for with various kinds of need pregnant women. Prefectural health centers expected the universal (population) approach to be widely applied for the support of pregnant women by the municipalities. On the other hand, prefectural health centers reported that home visits should be made by prefectural health centers for women with men- 
Table 2. Relationship between prefectural health centers' understanding of postpartum mental health screening results and their provision of psychiatric support.

\begin{tabular}{cccc}
\hline & & & $\mathrm{N}(\%)$ \\
\hline Cases & Understanding $(\mathrm{n}=128)$ & Not understanding $(\mathrm{n}=115)$ & Odds ratio 95\% CI \\
Requiring psychiatric support & $75(58.6)$ & $48(41.7)$ & 1.98 \\
Not requiring psychiatric support & $53(41.4)$ & $67(58.3)$ & $1.21-3.39$ \\
\hline
\end{tabular}

Table 3. Difference in methods of direct and indirect postpartum support provided by prefectural health centers depending on the understanding of mental health screening results.

\begin{tabular}{|c|c|c|c|c|c|}
\hline & & & & & n (\%) \\
\hline & Und & $n=136$ & Not un & $\lg n=127$ & $p$-value \\
\hline Telephone consultation & 55 & $(40.4)$ & 41 & $(32.3)$ & 0.2 \\
\hline Interview consultation & 54 & $(39.7)$ & 38 & $(29.9)$ & 0.12 \\
\hline Home visits & 73 & $(53.7)$ & 57 & $(44.9)$ & 0.17 \\
\hline Introduction to associated facilities ${ }^{1}$ & 48 & $(35.3)$ & 39 & $(30.7)$ & 0.43 \\
\hline Parenting group guidance & 31 & $(22.8)$ & 10 & (7.9) & $0.001^{*}$ \\
\hline Case conference & 41 & $(30.1)$ & 23 & $(18.1)$ & $0.031^{*}$ \\
\hline Network meeting & 9 & (6.6) & 3 & (2.4) & 0.14 \\
\hline Indirect support & & & & & \\
\hline Home visit by municipality's public health nurse & 83 & $(61.0)$ & 62 & $(48.8)$ & $0.04^{*}$ \\
\hline $\begin{array}{l}\text { Introduced municipality's public health nurse to associated } \\
\text { facilities }\end{array}$ & 53 & $(39.0)$ & 36 & $(28.3)$ & $0.09^{*}$ \\
\hline Explanation of the municipality's group guidance & 14 & $(10.3)$ & 5 & (3.9) & $0.05^{*}$ \\
\hline Explanation of the municipality's case conference & 49 & $(36.0)$ & 26 & $(20.5)$ & $0.006^{*}$ \\
\hline Explanation of the municipality's network meeting & 62 & $(45.6)$ & 33 & $(26.0)$ & $0.001^{*}$ \\
\hline
\end{tabular}

${ }^{1}$ Child abuse consultation center, Hospitals, School etc; Fisher's exact test ${ }^{*} \mathrm{P}<0.05$.

tal disorders, pregnant women with a history of abusing their first child, and pregnant women who have suffered child abuse. Concerning child abuse, prefectural health centers should support higher-risk mothers. Municipalities have the role of providing primary care, while prefectural health centers are responsible for secondary care, like acute care hospitals. Although the role is divided, it is desirable that prefectural health centers and municipalities cooperate and provide continuous support from pregnancy to postpartum period.

\subsection{Support for Unwanted Pregnancies}

According to reports of fatal cases of child abuse, unwanted pregnancy was a high-risk factor, but consultation services are only provided by $20 \%$ of prefectural health centers. The prefectural health centers that conducted such consultation services performed direct support, such as introducing pregnant women to associated facilities, conducting case conferences and conducting network meetings. Various kinds of indirect support were also conducted. Prefectural health centers that performed consultation services for pregnant women with unwanted pregnancies recognized the need to support high-risk pregnancies. Therefore, systems in which prefectural health centers cooperated with facilities and resources were established. However, these consultation services were not associated with postpartum support. In the future, it will be necessary to promote consultation services and a system in connection with postpartum support.

\subsection{Postpartum Support}

Approximately $70 \%$ of prefectural health centers conducted support for difficult postpartum cases. More support for difficult postpartum cases was conducted than support for difficult cases during pregnancy. Through the introduction of the universal (population) home visits of infants service project (KonnichiwaAkachan-Gijyo) starting in 2007, it is thought that the understanding of difficult postpartum cases may have become more common and straightforward. Home visits 
were the most common form of direct postpartum support provided by prefectural health centers, and the strategy for delivering support was the same as that for direct support for pregnant women. Subjects for whom prefectural health centers considered home visits from prefectural health centers to be more suitable than from municipalities for postpartum support included women with mental disorders, low-birth weight infants, women with a history of abusing their first child, and women with postpartum depression. These subjects were the same as those correlated with a high risk for child abuse. The prefectural health centers recognized mothers at high risk for child abuse to be candidates for support. Postpartum home visits by municipalities were considered a universal (population)-based approach, and postpartum home visits by prefectural health centers were considered approaches for high-risk families and individuals. Therefore, the current study identified a distinction between the different support roles for high- and low-risk subjects.

\subsection{Postpartum Mental Health Screening}

Among prefectural health centers in Japan, approximately $40 \%$ taught postpartum mental health screening to the municipalities using a specific method [26].

In order to effectively and impartially support subjects, a structured and concrete approach to screening for risk factors is a useful method for the prevention of abuse [27]. In addition, it is a standard component of childrearing to perform screening of the maternal field [28]. Prefectural health centers teach structured screening as a universal (population) approach, and it is necessary to fully understand the results. It is important that related institutions understand the results of the screening. It is also important that each institution identify high-risk pregnancies and evaluate the severity of the risk in each case. At the prefectural health centers that understood the results of the mental health screening, psychiatric consultation was understood to be important twice at the prefectural health centers that did not understand the results of mental health screening. It is very important to diagnose and support postpartum depression and postpartum psychosis early.

Prefectural health centers that understood the results of the mental health screening performed child-rearing group guidance and held case conferences more often than those that did not understand the results of the mental health screening. In addition, prefectural health centers that understood the results had concrete strategies for difficult postpartum cases and various types of indirect support. However, cooperation with municipalities remains a problem as only about half of prefectural health centers understood the screening results.

Approximately $30 \%$ of prefectural health centers taught the use of the 3-set questionnaire suggested by the Yo- shida group. In this questionnaire, Edinburgh Post-partum Depression Scale is used to screen women for postpartum depression. Risk factors for abuse are screened from a child-rearing support checklist. Extraction of the risk factors that lead to abuse is possible using a Mother-toInfant Bonding Scale. It is important to judge the risk to children while evaluating the psychiatric aspects of mothers [29]. Therefore, the 3-set questionnaire from the Yoshida group is useful for comprehensive judgment.

\section{LIMITATIONS}

The present study was limited to the analysis of prefecture health centers. While information about municipality health centers was not analyzed in the present study, that information can be used by the prefectural health centers to improve child-abuse prevention initiatives. Actions concerning local overall abuse prevention from both institutions should be evaluated in future studies. These issues remained, but this is a nationwide survey with a fairly high collection rate. Therefore it is very likely that the results reflected the Japanese overall situation.

\section{CONCLUSIONS}

This study contributes to child abuse prevention in the field of community maternal and child mental health. The purpose of this paper was to clarify the following issues through a Japanese nationwide survey of prefectural health centers, which are responsible for providing guidance to municipalities. And results indicated that an effective approach to the prevention of child abuse incorporates support systems for firstly perinatal mental health, secondly, unwanted pregnancies and lastly postpartum depression.

1) Prefectural health centers provide various kinds of direct or indirect support to municipalities. Home visits by municipalities were considered as a universal (population)-based approach, and home visits by prefectural health centers were considered as an approach for highrisk families and mothers.

2) Prefectural health centers that performed consultation services for pregnant women with unwanted pregnancies recognized the need to support high-risk pregnancies.

3) Psychiatric consultations were reported twice as often at the prefectural health centers that understood the results of the mental health screening compared to the centers that did not understand the results of mental health screening.

\section{ACKNOWLEDGEMENTS}

We are very grateful to the prefectural health centers for participating in this study. This study was supported by the Japanese Ministry of 
Health, Labour and Welfare and the Foundation for Children's Future (Research on Service for Children; Chief organizer: Dr. Kamibeppu).

\section{REFERENCES}

[1] Ministry of Health, Labor and Welfare (2007) An intermediate evaluation of the plan. Journal of Health and Welfare Statistics, 94, Tokyo. http://www.mhlw.go.jp/shingi/2006/03/s0316-4.html

[2] Ministry of Health, Labor and Welfare (2007) The verification results of the death caused by child abuse cases. http://www.mhlw.go.jp/houdou/2007/06/dl/h0622-5b.pdf

[3] Olds, D.L., Henderson, C.R., Chamberlin Jr., R., et al. (1986) Preventing child abuse and neglect: A randomized trial of nurse home visitation. Pediatrics, 78, 65-78.

[4] Olds, D.L., Eckenrode, J., Henderson, C.R., et al. (1997) Long-term effect of home visitation on maternal life course and child abuse and neglect: 15-year follow-up of randomized trial. Journal of the American Medical Association, 278, 637-643. doi:10.1001/jama.1997.03550080047038

[5] Donelan, M.N., Eckenrode, J. and Olds, D.L. (2009) Home visiting for the prevention of child maltreatment: Lessons learned during the past 20 years. Pediatrics Clinics of North America, 56, 389-403. doi:10.1016/j.pcl.2009.01.002

[6] MacMillan, H.L., Thomas, B.H., Jamieson, E., et al. (2005) Effectiveness of home visitation by public-health nurses in prevention of the recurrence of child physical abuse and neglect: A controlled trial. Lancet, 365, 1786-1793. doi:10.1016/50140-6736(05)66388-X

[7] Krugman, S.D., Lane, W.G. and Walsh, C.M. (2007) Update on child abuse prevention. General Pediatrics, 19, 711-718. doi:10.1097/MOP.0b013e3282f1c7e1

[8] Kitamura, T., Shima, S., Sugawara, M., et al. (1996) Clinical and psychosocial correlates of antenatal depression: A review. Psychotherapy and Psychosomatics, 65, 117123. doi: $10.1159 / 000289062$

[9] Kitamura, T., Yoshida, K., Okano, T., et al. (2006) Multicenter prospective study of perinatal depression in Japan: Incidence and correlates of antenatal and postnatal depression. Archives of Women's Mental Health, 9, 121-130. doi:10.1007/s00737-006-0122-3

[10] Honjyo, S., Arai, S., Kaneko, H., et al. (2003) Antenatal depression and maternal-fetal attachment. Psychopathology, 3, 304-311. doi:10:1159/000075189

[11] Okamoto, R. (2009) Community child health care in Japan. Journal of Family Health Care, 19, 210-211.

[12] Yamada, K. and Noda, J. (2002) A study on child abuse and neglect cared by public health nurse (PHN) of local health center: Based on nation wide survey. The Journal of Child Health, 61, 568-576.

[13] Kogure, M., Hasegawa, K., Osawa, M., et al. (2005) Collaborations of public health care facilities for supporting a family with a premature baby. Gunmakenrituiroyo Tankidaigakukiyo, 12, 69-81.

[14] Watanabe, Y. (2007) Perinatal medical care and child abuse prevention. Japanese Journal of Pediatrics, 60,
791-796.

[15] Nakaita, I. (2002) Development of the action for child abuse prevention. The Journal of Public Health Practice, 66, 531-533.

[16] Sato, A., Kitamiya, C., Lee, S.Y., et al. (2008) Circumstances and comparisons of child rearing burnout scales between neonatal mothers with and without home visit nursing. Japanese Journal of Public Health, 55, 318-325.

[17] Ueno, M. and Yamada, K. (1997) The role of public health nurses in child abuse and neglect. Bulletin of Osaka Prefecture College of Nursing, 3, 15-25.

[18] Ministry of Health, Labor and Welfare Statics Association (2007) Kokumin Eisei no Doukou. Ministry of Health, Labor and Welfare, 54, Tokyo.

[19] Ministry of Health, Labor and Welfare Statics Association (2007) Kokumin Fukushi no Doukou. Ministry of Health, Labor and Welfare, 54, Tokyo.

[20] Mother's \& Children's Health Organization (2009) Maternal and child health statistics of Japan. Wagakuni no Boshi Hoken, Tokyo.

[21] Mother's \& Children's Health Organization (2007) Maternal and child health statistics of Japan. Boshihoken no Omonaru Tokei, Tokyo.

[22] Ministry of Health, Labor and Welfare (2005) How to intervene the child abuse and neglect cases. Kodomo Gyakutai Taio no Tebiki, Tokyo.

[23] Yamashita, H. and Yoshida, K. (2004) Investigation of community based preventive intervention using the self report questionnaires for mothers at risk for child abuse: Contribution of perinatal psychiatry to child abuse in infancy. Japanese Journal of Child Abuse and Neglect, 6, 218-231.

[24] Cox, J.L., Holden, J.M. and Sagovsy, R. (1987) Detection of postnatal depression: Development of the 10-item Edinburgh Postnatal Depression Scale. British Journal of Psychiatry, 150, 782-786. doi:10.1192/bjp.150.6.782

[25] Yoshida, K., Yamashita, H., Conry, S., et al. (2012) A Japanese version of mother-to-infant bonding scale: Factor structure, longitudinal changes and links with maternal mood during the early postnatal period in Japanese mothers. Archives of Women's Mental Health, 15, 343352. doi:10.1007/s00737-012-0291-1

[26] Kamibeppu, K. (2008) Systems for perinatal mental health and child rearing. The Foundation for Children's Future, Tokyo.

[27] Olds, D.L. (2002) Prenatal and infancy home visiting by nurse: From randomized trials to community replication. Prevention Science, 3, 153-172. doi:10.1542/peds.110.3.486

[28] Flynn, L., Budd, M. and Modelski, J. (2008) Enhancing resource utilization among pregnant adolescents. Public Health Nursing, 25, 140-148. doi:10.1111/ j.1525-1446.2008.00690.x.

[29] Sato, T. (2008) Evaluation of a risk assessment scale for child abuse in the field of public health and development of systematic community public health activities for abuse prevention. Japanese Journal of Child Abuse and Neglect, 10, 66-74. 